\title{
Paper Clinical Value of Color Doppler Ultrasonography in Diagnosis of Lower Extremity Arterial Disease of Diabetes Patients
}

\author{
Jianyu Zhang1, Haijiao Mao² \\ ${ }^{1}$ Doppler Ultrasonic Department, Shaoxing Central Hospital, Shaoxing, China \\ ${ }^{2}$ Radiological Department, Qixian Branch of Shaoxing Central Hospital, Shaoxing, China \\ Email: 93957983@qq.com, 873818878@qq.com
}

How to cite this paper: Zhang, J.Y. and Mao, H.J. (2020) Paper Clinical Value of Color Doppler Ultrasonography in Diagnosis of Lower Extremity Arterial Disease of Diabetes Patients. Journal of Biosciences and Medicines, 8, 209-214.

https://doi.org/10.4236/jbm.2020.86020

Received: April 27, 2020

Accepted: June 27, 2020

Published: June 30, 2020

\begin{abstract}
Objective: To evaluate the diagnostic value of color Doppler ultrasound in the diagnosis of diabetic lower extremity vascular diseases. Methods: 48 patients with diabetic lower extremity vascular disease admitted in our hospital from September 2018 to September 2019 were included in the study and divided into the observation group, and another 48 patients with the same period of health examination in our hospital were included in the study and divided into the control group. Both groups used color Doppler ultrasound to detect the blood flow of lower extremity vessels. The incidence of blood flow, vascular diameter and stenosis, occlusion and arteriosclerosis of the lower extremity were observed. Results: The blood flow and vascular diameter of the lower extremity in the observation group were significantly lower than those in the control group $(\mathrm{p}<0.05)$. The incidence of vascular stenosis, vascular occlusion, thrombus, intimal thickening and plaque in the observation group was $85.42 \%, 22.92 \%, 10.42 \%$ and $93.75 \%$ respectively, which was significantly higher than that in the control group was $10.42 \%, 0.00 \%, 0.00 \%$ and $14.58 \%$ $(\mathrm{p}<0.05)$. The incidence of lower extremity vascular lesions in the observation group was significantly higher than that in the control group $(\mathrm{p}<0.05)$. Conclusion: Color Doppler ultrasound is of high diagnostic value in the diagnosis of diabetic lower extremity vascular diseases, and can be used to determine the blood flow of the lower extremity and the inner diameter of popliteal artery, thigh artery, dorsalis pedis artery and so on. At the same time, it can also clearly show the specific situation of vascular occlusion, arteriosclerosis and thrombosis, which is of great significance for the prevention and diagnosis of lower extremity vascular lesions, and can provide the basis for the treatment of lower extremity vascular lesions.
\end{abstract}




\section{Keywords}

Super Color Doppler Ultrasound, Diabetic Complications, Lower Extremity Vascular Disease, Diagnostic Value

\section{Introduction}

Diabetes is one of the most common chronic diseases with a high incidence in China, mainly among the middle-aged and the elderly [1]. Diabetic patients mainly present with elevated blood sugar. If their blood sugar is not controlled effectively, it may induce other diseases, especially various cardiovascular and cerebrovascular diseases, resulting in atherosclerosis. Lower extremity arterial disease is a common complication in patients with diabetes. Patients will suffer from lower extremity pain, limp, numbness, stiffness and other symptoms after onset. If not diagnosed and treated timely, it may even cause ischemic gangrene and amputation with the progression. Therefore, early diagnosis and treatment of lower extremity arterial disease are particularly important [2]. The symptoms of lower extremity arterial disease at the early stage are not obvious without any specific symptoms, but they can be diagnosed by color Doppler ultrasound, which has the advantages of non-invasiveness, simple operation, good economy and good repeatability. It has clinic value for diagnosing lower extremity arterial disease. This study aims to analyze the clinical value of color Doppler Ultrasound in the diagnosis of diabetic lower extremity arterial disease.

\section{Materials and Methods}

\subsection{Clinical Materials}

This study has been approved by the Ethics Committee. From September 2018 to September 2019, a total of 48 patients with lower extremity arterial disease admitted to our hospital were enrolled and typed into the observation group, and another 48 healthy people in the same period were typed into the control group. In the observation group, there were 26 males and 22 females, aged from 31 - 70 years (mean: $51.62 \pm 8.29$ years), course of disease 1 - 19 years (mean $(8.62 \pm 4.13)$ years $)$. In the control group, there were 25 males and 23 females, aged from 28 - 70 years (mean: $50.53 \pm 8.46$ years), with normal blood sugar. There was no significant difference in gender and age between the two groups ( $P>0.05)$, which was comparable. Inclusion criteria: 1) Patients who were informed and voluntarily participated in the study and signed informed consent; 2) Patients in the observation group who met the diagnostic criteria for type 2 diabetes in the "Guidelines for the Management of Type 2 Diabetes in China (Edition: 2017)" [3], and accompanied by symptoms of vascular diseases such as slight cooling, pain, numbness, stiffness of lower extremity, etc.; 3) Subjects whose diagnosis and clinical materials are kept intact. Exclusion criteria: 1) $\mathrm{Pa}$ tients with lower extremity arterial disease caused by other diseases; 2) Subjects 
with low compliance.

\subsection{Methods}

Both groups were examined and diagnosed by the same group of radiologists. The full-digital color Doppler diagnostic apparatus, Sonoline G50SonolineG50 of Siemens was applied in this study. Before the examination starts, adjust the relevant parameters, adjust the probe frequency to $7-12 \mathrm{MHz}$, sample volume 2 $\mathrm{mm}$, angle between sound beam and blood flow $<55$, and minimum flow velocity $10 \mathrm{~cm} / \mathrm{s}$. Lying in the supine position, the examinee were asked to bend and extend their lower extremity outward. The blood vessels of the lower extremity were examined by longitudinal cutting, mainly including superficial femoral artery, deep femoral artery, common femoral artery, anterior tibial artery, posterior tibial artery, popliteal artery, dorsalis pedis artery, etc. The vascular lumen was examined by crosscutting. In addition, the thickness of the inner wall during vasodilation was measured.

\subsection{Indicators for Observation}

The lower extremity blood flow and vessel diameter were compared between the two groups.

The angiostenosis, vascular occlusion, thrombosis, intimal thickening and plaque were compared between the two groups.

The incidence of pathological changes was compared between the two groups. Standard of vascular lesions: normal: the inner wall of blood vessel is smooth, blood flow and ultrasonic spectrum is normal. Lesions: the inner wall of the blood vessel is thickened, the blood flow is reduced, the blood flow signal is weakened, atherosclerosis and occlusion occurs in blood vessels, and the thickness of the vessel wall is $\geq 1 \mathrm{~mm}$ [4].

\subsection{Statistical Analysis}

The statistical analysis was performed by using SPSS version 20.0. The quantitative data were displayed as mean \pm standard deviation $( \pm \mathrm{s})$. T-test was used for the comparison of quantitative data. The counting data was expressed by $\%$ and chi-square test was used for the comparison of counting data. $\mathrm{P}<0.05$ was defined as statistically significant.

\section{Results}

\subsection{Blood Flow and Vascular Diameter of Lower Extremity}

Blood flow and vascular diameter in the observation group were significantly lower than those in the control group $(\mathrm{p}<0.05)$. See Table 1 for details.

\subsection{Changes of Lower Extremity Artery}

The incidence rates of vascular stenosis, vascular occlusion, thrombosis, intimal thickening and plaque in the observation group were $85.42 \%, 22.92 \%, 10.42 \%$ 
Table 1. Comparison of the blood flow and vascular diameter between two groups $(\bar{x} \pm s)$.

\begin{tabular}{ccccccc}
\hline & \multicolumn{2}{c}{ Popliteal Artery } & \multicolumn{2}{c}{ Femoral Artery } & \multicolumn{2}{c}{ Dorsalis Pedis Artery } \\
\cline { 2 - 7 } Group & $\begin{array}{c}\text { Blood flow } \\
\left(\mathrm{mm}^{3} / \mathrm{s}\right)\end{array}$ & $\begin{array}{c}\text { Vascular } \\
\text { diameter } \\
(\mathrm{mm})\end{array}$ & $\begin{array}{c}\text { Blood flow } \\
\left(\mathrm{mm}^{3} / \mathrm{s}\right)\end{array}$ & $\begin{array}{c}\text { Vascular } \\
\text { diameter } \\
(\mathrm{mm})\end{array}$ & $\begin{array}{c}\text { Blood flow } \\
\left(\mathrm{mm}^{3} / \mathrm{s}\right)\end{array}$ & $\begin{array}{c}\text { Vascular } \\
\text { diameter } \\
(\mathrm{mm})\end{array}$ \\
\hline $\begin{array}{c}\text { Observation } \\
\text { group (n = 48) }\end{array}$ & $10.62 \pm 2.14$ & $4.85 \pm 0.48$ & $35.18 \pm 4.39$ & $6.14 \pm 0.34$ & $0.75 \pm 0.37$ & $1.46 \pm 0.34$ \\
$\begin{array}{c}\text { Control group } \\
(\mathrm{n}=48)\end{array}$ & $13.83 \pm 3.28$ & $5.94 \pm 0.56$ & $43.17 \pm 5.58$ & $7.49 \pm 0.48$ & $1.49 \pm 0.64$ & $2.05 \pm 0.39$ \\
$\mathrm{t}$ & 5.856 & 10.239 & 7.797 & 15.907 & 6.935 & 7.900 \\
$\mathrm{P}$ & 0.000 & 0.000 & 0.000 & 0.00 & 0.000 & 0.000 \\
\hline
\end{tabular}

and $93.75 \%$ respectively, which were significantly higher than those in the control group (10.42\%, 0.00\%, 0.00\% and 14.58\%) $(\mathrm{p}<0.05)$. See Table 2 .

\subsection{Incidence Rates of Pathological Changes of Lower Extremity Artery}

The rate of pathological changes of the lower extremity artery in the observation group was significantly higher than that in the control group $(\mathrm{p}<0.05)$, as shown in Table 3.

\section{Discussion}

Diabetes is a chronic disease with a high incidence in China. As the material conditions of human life have been improved significantly, dietary structure has changed a lot, thus increasing the incidence rate of diabetes. This disease frequently occurs at younger age, seriously affecting the life quality of Chinese residents [5]. Diabetes patients with unsatisfactory blood sugar control and long-term hyperglycemia, are prone to have various complications, among which lower extremity arterial disease are one of the common complications. After the onset of the disease, irregular distortion and hardening of vascular intima occur in patients. If they do not receive timely treatment and intervention, necrosis of pathological tissues will also occur as the disease progresses, resulting in amputation or other serious adverse events [6]. Therefore, early diagnosis of lower extremity arterial disease has important clinical significance for the physical health of diabetes patients. In clinical practice, there exist many methods for the diagnosis of lower extremity arterial disease, including angiography and $\mathrm{X}$-ray. But due to their high cost and the potential damage they may bring to the body, the acceptance in patients is relatively low. In contrast, the Ultrasound has some advantages in the diagnosis of diabetic lower extremity arterial disease. Due to its non-invasiveness, good economy, it can be easily accepted by patients [7]. However, the diagnostic value of ultrasound still needs to be further studied. Color Doppler ultrasound can detect the location of vascular lesions by scanning longitudinal cutting and crosscutting when diagnosing vascular lesions of the 
Table 2. Comparison of changes of lower extremity artery between two groups.

\begin{tabular}{cccccc}
\hline Group & $\mathrm{N}$ & Hemadostenosis & $\begin{array}{c}\text { Vascular } \\
\text { Occlusion }\end{array}$ & Thrombosis & $\begin{array}{c}\text { Intimal Thickening } \\
\text { and Plaque }\end{array}$ \\
\hline $\begin{array}{c}\text { Observation } \\
\text { group }\end{array}$ & 48 & $41(85.42)$ & $11(22.92)$ & $5(10.42)$ & $45(93.75)$ \\
$\begin{array}{c}\text { Control group } \\
\mathrm{X}^{2}\end{array}$ & 48 & $5(10.42)$ & $0(0.00)$ & $0(0.00)$ & $7(14.58)$ \\
$\mathrm{P}$ & & 112.695 & 28.887 & 10.993 & 126.234 \\
\hline
\end{tabular}

Table 3. Comparison of rates of pathological changes in lower extremity artery between two groups.

\begin{tabular}{cccc}
\hline Group & $\mathrm{N}$ & $\begin{array}{c}\text { Patients with pathological } \\
\text { changes (n) }\end{array}$ & Incidence (\%) \\
\hline Observation group & 48 & 46 & 95.83 \\
Control group & 48 & 8 & 16.67 \\
$\mathrm{X}^{2}$ & & & 127.315 \\
$\mathrm{P}$ & & 0.000 \\
\hline
\end{tabular}

lower extremity. In addition, it can clearly determine the degree of stenosis and occlusion of vascular lumen, and can also observe information such as blood flow signal and blood volume trend. Patients with lower extremity arterial disease mainly present with arterial intimal thickening, enhanced echo and poor continuity of arterial intima at the lesions. When blood flows through the stenosis, blood flow signals will weaken and blood flow will decrease. When the vascular lumen is completely occluded, blood flow signals will disappear [8].

In the observation group, blood flow and vascular diameter in popliteal artery, femoral artery and dorsalis pedis artery were significantly lower than those of the control group. The main reason is that compared with the healthy people, patients with vascular diseases have narrower internal diameter and lower blood flow. The results of this study are consistent with the expected results, which is fully evidenced that the internal diameter of blood vessels and blood flow of patients with lower extremity arterial disease can be detected by ultrasonic examination, thus confirming the disease and guiding the prevention and treatment of the disease. In addition, the study also compared incidence rates of arteriosclerosis, vascular occlusion, thrombosis and vascular stenosis between the two groups. It showed that the incidence rate of the observation group was higher than that of the control group ( $\mathrm{p}<0.05$ ), which suggested that atherosclerosis, vascular occlusion, vascular stenosis and thrombosis could be found and diagnosed by ultrasound, and it was beneficial for the prevention and treatment of lower extremity arterial disease. In addition, the detection rate of ultrasound for vascular diseases was also compared. The diagnostic rate of ultrasound for vascular diseases was $95.83 \%$, which was at a high level and could meet the need of diagnosis. 
To sum up, the diagnosis of lower extremity arterial disease of diabetes patients by color Doppler ultrasound can reveal the pathological changes of blood vessels as early as possible, and can accurately display the blood flow and internal diameter of blood vessels, thus diagnosing and evaluating the specific conditions of patients, and providing corresponding diagnostic basis for symptomatic treatment.

\section{Conflicts of Interest}

The authors declare no conflicts of interest regarding the publication of this paper.

\section{References}

[1] Gu, S. (2017) Clinical Value of Color Doppler Ultrasound in Diagnosis of Diabetic Lower Extremity Arterial Disease. Guide of China Medicine, 15, 73-74.

[2] Wang, S., Liu, Y. and Tao, S. (2017) Clinical Effect of Color Doppler Ultrasound in Diagnosis of Diabetic Lower Extremity Arterial Disease. China Continuing Medical Education, 44, 22-23.

[3] Yi, J. (2017) Study on the Clinical Effect of Color Doppler Ultrasound in Diagnosing Diabetic Lower Extremity Arterial Disease. Imaging Research and Medical Application, 1, 51-52.

[4] Zeng, L., Zhao, P., Wan, R., et al. (2019) Diagnostic Value of Color Doppler Ultrasound in Lower Extremity Arteriosclerosis of Diabetes 2 Complicated with Subclinical Hypothyroidism. Chinese Journal of Ultrasound in Medicine, 35, 67-70.

[5] Li, J. (2018) Application Value of Color Doppler Ultrasound in Diagnosis of Lower Extremity Atherosclerosis. Imaging Research and Medical Application, 2, 106-107.

[6] Qiu, P. (2017) Diagnostic Value of Color Doppler Ultrasound in Lower Extremity Arterial Disease of Diabetic Patients. Chinese Journal of Practical Medical Imaging, 18, 393-395.

[7] Huang, J. and Tang, H. (2017) Analysis on the Color Doppler Ultrasound Diagnosis of Lower Extremity Arterial Disease in Diabetes 2 and Related Factors. Shenzhen Journal of Integrated Traditional Chinese and Western Medicine, 27, 66-67.

[8] Zhao, G. (2017) Diagnostic Value of Color Doppler Ultrasound on Lower Extremity Arterial Disease of Diabetes 2. Shenzhen Journal of Integrated Traditional Chinese and Western Medicine, 27, 98-100. 